Research Article

Animal Genetics

\title{
Characterization of three new mitochondrial genomes of Coraciiformes (Megaceryle lugubris, Alcedo atthis, Halcyon smyrnensis) and insights into their phylogenetics
}

\author{
Meidong Jing ${ }^{1 *}$, Huanhuan Yang ${ }^{2 *}, \mathrm{Kai}^{3}{ }^{3}$ and Ling Huang ${ }^{1}$ ic \\ ${ }^{I}$ Nantong University, School of Life Sciences, Nantong, Jiangsu, P. R. China. \\ ${ }^{2}$ Ludong University, School of Life Sciences, Yantai, Shandong, P. R. China. \\ ${ }^{3}$ Nantong Xingdong International Airport, Nantong, Jiangsu, P. R. China.
}

\begin{abstract}
Coraciiformes contains more than 200 species with great differences on external morphology and life-style. The evolutionary relationships within Coraciiformes and the phylogenetic placement of Coraciiformes in Aves are still questioned. Mitochondrial genome (mitogenome) sequences are popular markers in molecular phylogenetic studies of birds. This study presented the genome characteristics of three new mitogenomes in Coraciiformes and explored the phylogenetic relationships among Coraciiformes and other five related orders with mitogenome data of 30 species. The sizes of three mitogenomes were 17,383 bp (Alcedo atthis), 17,892 bp (Halcyon smyrnensis) and 17,223 bp (Megaceryle lugubris). Each mitogenome contained one control region and 37 genes that were common in vertebrate mitogenomes. The organization of three mitogenomes was identical to the putative ancestral gene order in Aves. Among 13 available Coraciiform mitogenomes, 12 protein coding genes showed indications of negative selection, while the MT-ND6 presented sign of positive selection or relaxed purifying selection. The phylogenetic results supported that Upupidae and Bucerotidae should be separated from Coraciiformes, and that Coraciiformes is more closely related to Piciformes than to Strigiformes, Trogoniformes and Cuculiformes. Our study provide valuable data for further phylogenetic investigation of Coraciiformes.
\end{abstract}

Keywords: Mitogenome, Alcedo atthis, Halcyon smyrnensis, Megaceryle lugubris, phylogeny.

Received: November 22, 2019; Accepted: July 13, 2020.

\section{Introduction}

Coraciiformes consists of more than 200 species with great differences in distribution region, body size, external morphology and life-style. There are 25 Coraciiform species in China, among which the largest species (Buceros bicornis) is about three to four kilograms in weight and 119 to1 28 centimeters in body length. However, the smallest species (Alcedo atthis) is only about 30 to 40 grams in weight and 150 to 170 millimeters in length (Zhao, 2001). The habitat and diet of Coraciiform species are also diverse. Some species (Alcedinidae) live around rivers and lakes, and feed on fish and shrimps; some species (Bucerotidae, Meropidae, Upupidae) live in forests and feed on fruits, seeds or insects; other species (Coraciidae) live in the plain and feed on insects and small animals (Zhao, 2001).

In classical taxonomy, Coraciiformes included 10 families (Alcedinidae, Bucerotidae, Brachypteracidae, Coraciidae, Leptosomatidae, Meropidae, Momotidae, Phoeniculidae, Todidae, and Upupidae) (Wetmore, 1960).

Send correspondence to Ling Huang. Nantong University, School of Life Sciences, Nantong, Jiangsu, China. E-mail: huangdl@126.com.

*These authors contributed equally to this work.
However, later investigations based on anatomy of the feeding apparatus (Burton, 1984) and fossil records (Olson, 1985) proposed that the "Bucerotes" (Bucerotidae, Phoeniculidae and Upupidae) should be separated from Coraciiformes. The phylogenetic position of Leptosomatidae also should be questioned according to the analyses of different sets of morphological characters (Mayr et al., 2003; Mayr, 2005). In addition, different morphological assessments deduced inconsistent conclusions on the relationships among Coraciiformes and other related orders. For example, studies based on myological characters showed that Trogonidae (Trogoniformes) should be included in Coraciiformes (Cracraft, 1981; Maurer and Raikow, 1981), while investigations with osteological characters suggested a distant relationship between Trogonidae and Coraciiformes (Höfling and Alvarenga, 2001). Studies by Burton (1984) and Olson (1985) proposed that "Bucerotes" was more closely related to Piciformes than to other families in Coraciiformes, while analyses of 98 morphological characters suggested a closer relationship between Piciformes and classical Coraciiformes (Mayr, 2005).

Molecular phylogenetic investigations based on nuclear (Sibley and Ahlquist, 1990; Kemp, 1995; Johansson et al., 2001; Hackett et al., 2008; Jetz et al., 2012; Ödeen and 
Hästad, 2013; Jarvis et al., 2014; Prum et al., 2015; Reddy et al., 2017) or mitochondrial gene markers (de los Monteros, 2000; Ericson et al., 2006; Pacheco et al., 2011; Mahmood et al., 2014; Sun et al., 2017; Tamashiro et al., 2019) provided powerful evidence on the separated position of "Bucerotes". The separated position of Leptosomatidae was also supported by molecular phylogenetic investigations (Ericson et al., 2006; Hackett et al., 2008). However, most of above studies aimed to resolve the high-level phylogenetic relationships in Aves, species involved in these studies were limited, and the relationships among Coraciiformes and related orders were still in controversy. As for the relationship among Coraciiformes, "Bucerotes", Piciformes and Trogoniformes, some analyses using multiple nuclear genes (e.g., Hackett et al., 2008; Jetz et al., 2012; Ödeen and Hästad, 2013; Jarvis et al., 2014; Prum et al., 2015; Reddy et al., 2017) and mitogenome data (e.g., Mahmood et al., 2014; Tamashiro et al., 2019) supported the topology of (Trogoniformes, (Bucerotes, (Coraciiformes, Piciformes))), while some studies with mtDNA data indicated the topology of ((Coraciiformes, Bucerotes), (Piciformes, Trogoniformes))" (e.g. Pacheco et al., 2011) or (Piciformes, (Coraciiformes, (Trogoniformes, Bucerotes))) (de los Monteros, 2000).

Mitochondrial genomes (mitogenomes), because of their advantageous characteristics (small size, simple organization, lack of recombination, rapid nucleotide substitution), have been extensively applied in phylogenetic studies of birds since the report of chicken mitogenome (e.g. Desjardins et al., 1990; Cooper et al., 2001; Paton et al., 2002; Morgan-Richards et al., 2008; Pratt et al., 2009; Nabholz et al., 2010; Pacheco et al., 2011; Zhou et al., 2014; Bruxaux et al., 2018; Tamashiro et al., 2019). Some researchers proposed that the molecular studies using only mtDNA markers have limitations in testing phylogenetic hypotheses, because incomplete lineage sorting, adaptive introgression, demographic disparities or sex-biased asymmetries exist in many animal systems (Toews and Brelsford, 2012). However, phylogenies inferred from mitogenome data can complement and confirm the results based on nuclear gene markers, they are still essential in phylogenetic investigations. Up to date, mitogenomes of about 800 avian species have been released in GenBank, among which only 13 species belong to Coraciiformes (three species in this study were included). The accumulation of mitogenome data in Coraciiformes will be helpful to explore the phylogenetic puzzles on this order.

Here, we provide three new mitogenome data in Alcedinidae of Coraciiformes: Common Kingfisher (Alcedo atthis), White-throated Kingfisher (Halcyon smyrnensis) and Crested Kingfisher (Megaceryle lugubris). This study aims to 1) elucidate the structural characteristics of three mitogenomes and compare our data with other Coraciiform mitogenomes available in GenBank; 2) explore the phylogenetic relationships among 12 families of six orders (Coraciiformes, Piciformes, Strigiformes, Cuculiformes,
Trogoniformes, Psittaciformes) with mitogenome data of 30 species.

\section{Material and Methods}

\section{Genomic DNA preparation}

The samples of Megaceryle lugubris, Alcedo atthis and Halcyon smyrnensis were collected from Nantong national airport, Jiangsu Province, China. The identification of the specimens was according to external morphologies (Sibley and Monroe, 1990). The muscle tissues were preserved in absolute ethanol and were stored at $-80^{\circ} \mathrm{C}$. A Wizard Genomic DNA purification kit (Promega, Madison, WI, USA) was used to extract the total genomic DNA. Concentration of the genomic DNA was determined with a spectrophotometer and was adjusted to $50 \mathrm{ng} / \mu \mathrm{L}$.

\section{PCR amplification and sequencing}

To amplify overlapping segments spanning the whole mitogenome, 28 sets of primers reported by Sorenson et al. (1999) were used. The amplified segments were all smaller than $1,500 \mathrm{bp}$, and all segments overlapped each other by $200 \mathrm{bp}$. The amplifications were completed in a Mycycler Gradient thermocycler (Bio-Rad), and the volume of each reaction was about $50 \mu \mathrm{L}$, containing $25 \mu \mathrm{L}$ of Premix Taq (TaKaRa TaqTM Version 2.0 plus dye, Takara Biotechnology, Dalian, China), $1 \mu \mathrm{L}(20 \mu \mathrm{M})$ of each primer, $22.5 \mu \mathrm{L}$ of deionized water and $0.5 \mu \mathrm{L}$ of genomic DNA (about 25-30 ng). The PCR processes were consistent with those reported previously (Sun et al., 2017). To check for contamination, each round of PCR included a negative control (without genomic DNA), and there were no products in all negative controls. The PCR products were electrophoresed on $1.5 \%$ agarose gels staining with ethidium bromide, and were visualized by ultraviolet transillumination. The purification and sequencing of the PCR products were same with those described in Sun et al. (2017).

\section{Sequence assembly and gene annotation}

Sequence assembly and annotation were performed with DNASTAR package (Lasergene version 5.0; Madison, WI, USA). The boundaries of rRNA genes and protein coding genes (PCGs) were detected by aligning our sequences with other available Coraciiform mitogenomes in GenBank: Ceryle rudis (NC_024280), Halcyon pileata (NC_024198) (Sun et al., 2017), Halcyon coromanda (NC_028177) (Park et al., unpublished data), Todirhamphus sanctus (NC_011712) (Pratt et al., 2009), Aceros waldeni (NC_015085) (Sammler et al., 2011), Bycanistes brevis (NC_015201) (Pacheco et al., 2011), Penelopides panini (HQ834451) (Sammler et al., 2011), Eurystomus orientalis (NC_011716) (Pratt et al., 2009), Merops viridis (NC_034642) (Huang et al., 2017) and Upupa epops (NC_028178) (Park et al., unpublished). Gene annotations were conducted with the MITOS webserver (Bernt et al., 2013) and tRNAscan-SE 2.0 (http://lowelab.ucsc.edu/tRNAscan-SE/) (Lowe et al., 
2016). Cloverleaf secondary structure and anticodons of tRNA genes were determined with the web-server of the tRNAscan-SE v 2.0 (Lowe et al., 2016). The formulas AT skew $=[\mathrm{A}-\mathrm{T}] /[\mathrm{A}+\mathrm{T}]$ and $\mathrm{GC}$ skew $=[\mathrm{G}-\mathrm{C}] /[\mathrm{G}+\mathrm{C}]$ were used to calculate the skewness values (Junqueiraa et al., 2004). The relative synonymous codon usage (RSCU) values were measured by MEGA X 10.1 (64-bit) BETA (for Windows) program (Kumar et al., 2018).

\section{Phylogenetic relationships inferred from mitogenome data}

To investigate the phylogenetic relationships among Coraciiformes and other related orders that have been referred in previous studies (de los Monteros, 2000; Hackett et al., 2008; Pacheco et al., 2011; Jetz et al., 2012; Jarvis et al., 2014; Mahmood et al., 2014, Reddy et al., 2017), phylogenetic trees including 30 species belonging to six orders (12 families) were reconstructed with mitogenome sequences. Gallus gallus (NC_001323) was chosen as outgroup. The mitogenome information of all species involved were shown in Table S1.

Two different sets of data were used in phylogenetic analyses. In the first set, the control regions (CRs) of all mitogenomes were deleted, because the great differences in CRs would lead to stochastic errors or the effect of homoplasy. The second set of data included the sequences of 12 protein coding genes (PCGs). MT-ND6 was excluded because of the very different evolutionary tendency from other 12 PCGs based on the $\mathrm{dN} / \mathrm{dS}$ ratios.

MrBayes 3.2.7a (Ronquist and Huelsenbeck, 2003) was applied to construct the Bayesian tree. The program Modeltest version 3.7 (Posada and Crandall, 1998) chose the $\mathrm{GTR}+\mathrm{I}+\mathrm{G}$ model and the GTR $+\mathrm{G}$ model as the appropriate substitution model of sequence evolution for the first and second set of data, respectively. The detailed processes for Bayesian tree construction were the same as those reported previously (Sun et al., 2017). We used two independent runs to confirm the convergence of the Bayesian posterior probabilities (BPP) distribution.

\section{Results and Discussion}

\section{Mitogenome organization and nucleotide composition}

The mitogenomes of $A$. atthis, $H$. smyrnrnsis and $M$. lugubris are circular and double-stranded macromolecules, with the size of $17,383 \mathrm{bp}, 17,892 \mathrm{bp}$ and $17,223 \mathrm{bp}$, respectively (Table S2, S3). The accession numbers of the three mitogenomes in GenBank are NC_035868 (KY964271, A. atthis), NC_035746 (KY940559, H. smyrnensis) and NC_035658 (KY940558, M. lugubris). Among 13 available Coraciiform mitogenomes (Table 1), the smallest one is $16,542 \mathrm{bp}$ (H. coromanda), and the largest one is $22,737 \mathrm{bp}$ ( $P$. panini). Great variation in lengths of control regions (CRs) is the main reason for size difference of these mitogenomes (Table S3).

The mitogenomes of $A$. atthis, $H$. smyrnrnsis and $M$. lugubris contain 37 genes (including 22 tRNA genes, 13 PCGs and two rRNA genes) and one CR (Table S2). Twenty-eight genes (14 tRNA genes, 12 PCGs and two rRNA genes) are located on the $\mathrm{H}$ strand, and the remaining nine genes are located on the L strand (Table S2). All genes are compactly arranged, and gene overlaps exist at several gene junctions (Table S2). Gene arrangement of the three mitogenomes is identical to the putative ancestral gene order in avian mitogenomes (Gibb et al., 2007; Song et al., 2015). The comparative circular map is presented to visualize the genome organizations of 13 Coraciiform mitogenomes (Figure 1).

All 13 Coraciiform mitogenomes show nucleotide bias toward $\mathrm{A}+\mathrm{T}$ in both strands, and display slight positive AT-skews and negative GC-skews (Table 1, Table S3), which suggest that the content of adenine is higher than that of thymine, and the content of cytosine is higher than that of guanine. Except the MT-RNR1 in five species (A. waldeni,

Table 1 - Coraciiform species of mitogenomes analyzed in this study.

\begin{tabular}{|c|c|c|c|c|c|c|c|}
\hline Family & Species & Accession & Genome Size (bp) & $\mathrm{A}+\mathrm{T} \%$ & AT-skew & GC-skew & Reference \\
\hline \multirow{7}{*}{ Alcedinidae } & Alcedo atthis & NC_035868 & 17,383 & 55.3 & 0.085 & -0.383 & This study \\
\hline & Ceryle rudis & $\mathrm{NC}_{-} 024280$ & 17,355 & 55.9 & 0.148 & -0.400 & Sun et al. (2017) \\
\hline & Halcyon pileata & NC_024198 & 17,612 & 53.7 & 0.140 & -0.401 & Sun et al. (2017) \\
\hline & Halcyon smyrnensis & NC_035746 & 17,892 & 54.3 & 0.140 & -0.409 & This study \\
\hline & Halcyon coromanda & NC_028177 & 16,542 & 53.9 & 0.141 & -0.406 & DS \\
\hline & Megaceryle lugubris & NC_035658 & 17,223 & 55.1 & 0.143 & -0.384 & This study \\
\hline & Todiramphus sanctus & NC_011712 & 17,549 & 55.2 & 0.132 & -0.394 & Pratt et al. (2009) \\
\hline \multirow[t]{3}{*}{ Bucerotidae } & Aceros waldeni & NC_015085 & 21,657 & $55 . .0$ & 0.133 & -0.383 & Sammler et al. (2011) \\
\hline & Bycanistes brevis & NC_015201 & 17,591 & 53.0 & 0.137 & -0.377 & Pacheco et al. (2011) \\
\hline & Penelopides panini & NC_015087 & 22,737 & 55.2 & 0.136 & -0.396 & Sammler et al. (2011) \\
\hline Coraciidae & Eurystomus orientalis & NC_011716 & 17,210 & 53.5 & 0.127 & -0.400 & Pratt et al. (2009) \\
\hline Meropidae & Merops viridis & NC_034642 & 18,295 & 51.9 & 0.101 & -0.400 & Huang et al. (2017) \\
\hline Upupidae & Upupa epops & $\mathrm{NC} \_028178$ & 16,562 & 55.4 & 0.136 & -0.371 & DS \\
\hline
\end{tabular}




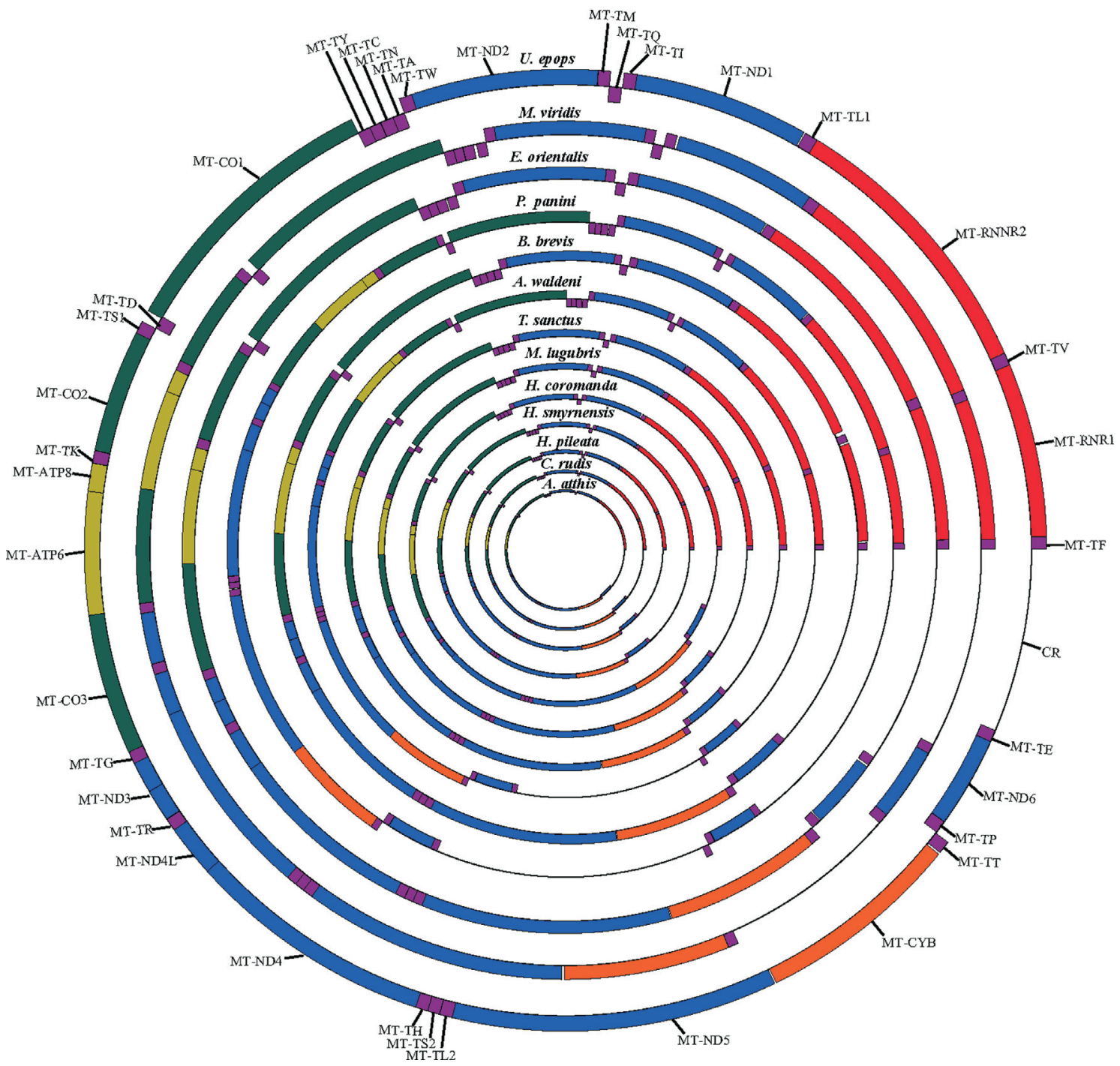

Figure 1 - Comparative circular map showing the genome organizations of 13 Coraciiform mitogenomes listed in Table 1.

B. brevis, $P$. panini, $M$. viridis, $U$. epop), other fragments in 13 mitogenomes present nucleotide bias toward $\mathrm{A}$ and $\mathrm{T}$. The $\mathrm{A}+\mathrm{T}$ bias in CRs are more significant (Table S3).

\section{Structure of control regions}

CRs of different species are highly variable and distinctive. The length of CR in hte mitogenomes of $A$. atthis, $H$. smyrnensis, and M. lugubris is $1,850 \mathrm{bp}, 2,333 \mathrm{bp}$ and $1,672 \mathrm{bp}$, respectively. Among 13 Coraciiform mitogenomes, the longest $\mathrm{CR}$ is $5,863 \mathrm{bp}$ in size ( $P$. panini) (Table S3).

In addition to great variation in length, complex rearrangements have happened around CRs and flanking genes in some species. To date, at least seven gene orders have been identified in avian mitogenomes: (1) ancestral avian CR; (2) duplicate CR; (3) duplicate TT-CR; (4) duplicate TT-TP and CR; (5) duplicate TE-CR; (6) remnant CR; (7) erroneous gene order (Desjardins and Morais, 1990; Mindell and Sorenson, 1998; Eberhard and Wright, 2001; Abbott et al., 2005; Gibb et al., 2007; Singh et al., 2008; Verkuil et al.,
2010; Zhou et al., 2014; Kang et al., 2018). Three types of gene orders were identified in 13 Coraciiform mitogenomes (Figure 1), with ancestral CR type for 10 mitogenomes, duplicate TT-CR for two mitogenomes $(A$. waldeni and $P$. panini), and duplicate CR for one mitogenome ( $M$. viridis).

Based on conserved motifs (Sbisà et al., 1997; Pratt and Gibb, 2009; Sammler et al., 2011; Park et al., 2015; Huang et al., 2017; Sun et al., 2017), the CRs of $A$. atthis, $H$. smyrnensis, and $M$. lugubris were divided into three different domains: the peripheral and highly variable domains (I and III), and the conserved domain II (Figure S1). In Domain I, there are two extended blocks (ETAS1-2) including sequences that are responsible for the termination of replication (TAS, 5'-TATAT-3' and 5'-TACAT-3') (Figure S1, Table S4). A CSBI-LIKE block (sequence that is similar to the conserved block) also exists in Domain I. There are seven conserved blocks (C, D, E, F, CSBa, b and B) in Domain II of H. smyrnensis and M. Lugubris, while B-box is absent in Domain II of $A$. atthis (Figure S1, Table S4). Domain III includes a conserved block (CBS1) that is responsi- 
ble for the regulation of mtDNA replication, a heavy strand replication origin $\left(\mathrm{O}_{\mathrm{H}}\right)$, bi-directional transcription promoter of L- and H- strand (LSP/HSP), and a poly (T) sequence at downstream of the CSB1 (Figure S1, Table S4). Besides, there are tandem repeats near the 3' terminal of Domain III. There are two types of repeats in A. atthis (5'-TTCGTTTG-3'

5'-ACAAAACAAACGAATCAATTAGACTTTATCTAC
-3') and $M$. lugubris (5'-CAATTAACGAA-3'; 5'-CATTAACGAA-3'), and three types of repeats in $H$. smyrnensis 5'-TCGTTGATCGAT-3'; 5'-CATAAATTCTGACAAATTAACGAATGAACTCTA ATTACACAAGCAGACATTCCCAACAAACAAAAT-3 '). The difference in repeat sequences lead to different size of CRs in three species. The origination and evolutionary history of different types of repeat sequences in CRs of animal mitogenomes have not been fully solved (Levinson et al., 1987; Piganeau et al., 2004; Mjelle et al., 2008; Leclercq et al., 2010; Sammler et al., 2011; Shi et al., 2013).

\section{Structure of transfer and ribosomal RNA genes}

All 22 tRNA genes that are common in vertebrate mitogenomes are found in the mitogenomes of $A$. atthis, $H$. smyrnensis, and $M$. lugubris (Figure 1). The lengths of these genes in three mitogenomes are similar (Table S2). All 22 tRNAs can fold into normal clover-leaf secondary structure (Figure S2). Apparent length difference and nucleotide variations exist in the TYC and DHU loops and stems, while the anticodon loop, anticodon stem, and acceptor stem are more conserved in length. Atypical pairings of G-U and unmatched base pairs of A-C, A-A, U-U, and U-C are scattered throughout the stems (Figure S2). These kinds of mismatched pairs or unmatched base pairs have also been found in other vertebrate and invertebrate mitogenomes (Harlid et al., 1998; Slack et al., 2003; Li et al., 2007; Bi et al., 2012; Zou et al., 2015; Sun et al., 2017; Bi et al., 2019; He et al., 2019).

Two rRNA genes locate between MT-L1 and MT-TF, and they are separated by MT-TV (Figure 1, Table S2). The lengths of the MT-RNR1 in 13 Coraciiform mitogenomes range from $966 \mathrm{bp}$ (M. lugubris) to $981 \mathrm{bp}$ (H. coromanda) (Table S3). The secondary structures of MT-RNR1 for $A$. atthis, H. Smyrnensis and M. lugubris are similar, comprising three main domains and 46 helices (Figure S3). Nucleotide substitutions are mainly located at H609, H768, H814 and loops near H307, H655, H683, and H884. The sizes of the MT-RNR2 in 13 Coraciiform mitogenomes range from $1,578 \mathrm{bp}$ (U. epops) to 1,614 bp (M. viridis) (Table S3). The secondary structures of MT-RNR2 for A. atthis, $H$. Smyrnensis and M. lugubris contain six domains and 59 helices (Figure S4). The sequences for Domain IV are conserved, while there are many nucleotide substitutions in Domain I-III, V and VI. The secondary structures of two rRNAs in many avian species were similar, while there were obvious differences in secondary structures of mitochondrial
rRNAs between birds and bees (De Los Monteros, 2003; Li et al., 2015; Bi et al., 2019; He et al., 2019; this study).

\section{Characteristics of Protein Coding Genes (PCGs)}

All 13 PCGs that are typical in animal mitogenomes are identified in the mitogenomes of $A$. atthis, $H$. smyrnensis and M. lugubris, among which only MT-ND6 is encoded by the L strand (Figure 1, Table S2). The lengths of each PCGs in the three mitogenomes are almost the same (Table S5). MT-ND5 (1,815 bp) is the longest one, and MT-ATP8 (168 bp) is the shortest one. Twelve PCGs show nucleotide bias toward A+T, while the MT-ND6 displays slight nucleotide bias toward $\mathrm{G}+\mathrm{C}$. Except the MT-ND1, MT-ND3, and MT-CYB of $A$. atthis, other PCGs show a positive AT skews and a great negative GC skew (Table S5). The AT skews for MT-ND6 are significantly greater than those for other PCGs.

ATN is the start codon of most PCGs in three Coraciiform mitogenomes, while the start codon of MT-COI is GTG, and the start codon for MT-ND3 of A. atthis is TAA (Table S2). Ten PCGs terminate with AGG, TAA, or TAG, and the remaining three PCGs (MT-CO3, MT-ND2, and MT-ND4) have incomplete stop codons (T), which can be adjusted to a TAA terminal codon by posttranscriptional polyadenylation (Ojala et al., 1981).

The nucleotide composition of MT-ND6 is very different from those of other PCGs, so it is not included in the codon usage analyses. Except the stop codons, 12 PCGs of the three Coraciiform mitogenomes contain 3,796 (A. atthis) and 3,793 (H. smyrnensis and M. lugubris) codons, respectively. The content of $\mathrm{A}+\mathrm{T}$ in all three codon positions are larger than or equal to $50 \%$. For other 10 Coraciiform mitogenomes, the content of $\mathrm{A}+\mathrm{T}$ in the second codon positions are greater than $50 \%$, whereas the nucleotide bias toward $\mathrm{A}+\mathrm{T}$ or $\mathrm{G}+\mathrm{C}$ in the first and third codon positions are varied in different species (Table S6). The G content in the second and third codons are low, especially, the G content in the third codons are only $3.6 \%-6.0 \%$ in 13 Coraciiform mitogenomes. The low $\mathrm{G}$ content in third codon positions of mitochondrial PCGs is a common feature in mammalian and avian mitogenomes (Harlid et al., 1998; Gibson et al., 2005; Zou et al., 2015; Gong et al., 2017; Sun et al., 2017; Bi et al., 2019). The variation ranges of nucleotide content in three codon positions are negatively correlated to the selective pressure that they are subjected to (Zhong et al., 2002). The second codon positions subject to the greatest selective pressure, corresponding to the smallest nucleotide variation range (Table S6).

Relative synonymous codon frequencies (RSCU) was applied to calculate the codon usage of 12 PCGs of the three Coraciiform mitogenomes, and the results are showed in Figure S5. Corresponding to the high content of A and C in the third codon positions (Table S6), the frequently used codons are NNA and NNC (Figure S5). Eight types of amino acids (L, V, S, P, T, A, R, and G) are frequently used, among which the leucine is the most frequently used amino acids.

The extra insertion of "C" in MT- ND3 (position 174) that was identified in some avian mitogenomes (Harlid et al., 
1998; Kan et al., 2010a, 2010b; Yang et al., 2010; Zhang et al., 2012; Ren et al., 2014; Sun et al., 2017; Bi et al., 2019) also exists in nine Coraciiform mitogenomes, including $A$. atthis, H. smyrnensis and M. lugubris (Figure S6).

\section{The evolutionary patterns of mitochondrial PCGs among 13 Coraciiform species}

The total length of aligned 13 mitochondrial PCGs from 13 Coraciiform species is 11,402 bp (without gaps), and there are 5,483 variable sites (Table 2). Based on the percent of variable sites, the most variable gene is MT-ATP8, followed by MT-ND6, MT-ND2, and MT-ND5. In contrary, the most conserved gene is MT-CO1, then MT-CO2 and MT-CO3 (Table 2). The nucleotide diversity ( $\pi$ ) of 13 PCGs varies from 0.145 (MT-CO1) to 0.233 (MT-ATP8). The $\pi$ value is positively correlated with the percent of variable sites. The ts/tv ratios of 13 PCGs range from 1.21 (MT-ND4L) to 2.72 (MT-ND6), and the $\mathrm{dN} / \mathrm{dS}$ ratios vary from 0.047 (MT-CO1) to 1.151 (MT-ND6) (Table 2). There was no correlation between the ts/tv and $\mathrm{dN} / \mathrm{dS}$ ratios.

Combining our results and previously published data (Liang et al., 2015; Bi et al., 2019), we could find some concordant features on the evolution of avian mitochondrial PCGs: 1) MT-ATP8 is the most diverse gene, while MT-CO1, MT-CO2 and MT-CO3 are very conserved; 2) most PCGs were subjected to negative selection, while the protein evolution rates of these genes vary greatly in different groups; 3) more nucleotide transitions than transversions have happened in all PCGs; 4) in different groups, the evolutionary patterns of the MT-ND6 are different. The dN/dS ratio of the MT-ND6 among Coraciiform species is greater than 1 (Table 2), indicating positive selection or relaxed purifying selection effect.

\section{Phylogenetic relationships among 30 species based on mitogenome data}

The relationships among Coraciiformes and several related orders were in debate both in morphological taxonomic studies (Cracraft, 1981, 1988; Burton, 1984; Höfling and Alvarenga, 2001; Mayr, 2003, 2005) and molecular phylogenetic studies using nuclear DNA (Hackett et al., 2008; Jetz et al., 2012; Jarvis et al., 2014; Reddy et al., 2017) and mtDNA (de los Monteros, 2000; Pacheco et al., 2011; Mahmood et al., 2014;). Biological factors such as incomplete lineage sorting, hybridization and adaptive introgression, demographic disparities and sex-biased asymmetries (Toews and Brelsford, 2012) and potential systematic errors in analysis methods (Brinkmann and Philippe, 2008) often lead to nuclear and mitochondrial phylogenetic discordance in many animal systems. Analyses with single genes would increase stochastic errors and the effect of homoplasy (Campbell and Lapointe 2011). Reconstructing phylogenetic trees with both mitochondrial and nuclear genome sequences is a new strategy to solve evolutionary puzzles in animals. Here, the mitogenome sequences of 30 species in 12 families are used to explore the phylogenetic placements of Coraciiformes and other five orders. G. gallus (NC_001323) is used as outgroup (Table S1).

The phylogenetic trees inferred from two sets of data have the same topologies (Figure 2). The topologies are also consistent with the results based on mitogenome data reported by Sun et al. (2017) though four new species were added in the present analyses. The monophyly of all 12 families are well supported, and they cluster into three distinct clades (Figure 2). Clade A consists of two subclades: one subclade contains species of Alcedinidae, Coraciidae and Meropidae; another subclade contains species of Bucerotidae, Upupidae, and Picidae. Clade B also comprises two subclades: one subclade includes species of Strigidae and Tytonidae; another subclade includes species of Cuculidae and Trogonidae. Clade A and clade B are sister

Table 2 - Rates and patterns of evolution among mitochondrial PCGs and 13 species of Coraciiformes.

\begin{tabular}{|c|c|c|c|c|c|c|c|}
\hline Gene & Length (bp) & Var. sites $(\%)$ & $\pi$ & $\mathrm{dN}$ & dS & $\mathrm{dN} / \mathrm{dS}$ & ts $/$ tv \\
\hline MT-ATP6 & 684 & $323(47.22 \%)$ & 0.180 & 0.061 & 0.480 & 0.128 & 1.27 \\
\hline MT-ATP8 & 168 & $100(59.52 \%)$ & 0.233 & 0.153 & 0.483 & 0.317 & 1.24 \\
\hline MT-CO1 & 1551 & $565(36.43 \%)$ & 0.145 & 0.023 & 0.488 & 0.047 & 1.72 \\
\hline MT-CO2 & 684 & $275(40.20 \%)$ & 0.154 & 0.045 & 0.480 & 0.094 & 1.57 \\
\hline MT-CO3 & 784 & $324(41.33 \%)$ & 0.151 & 0.047 & 0.460 & 0.103 & 1.62 \\
\hline MT-CYB & 1143 & $495(43.31 \%)$ & 0.172 & 0.062 & 0.479 & 0.129 & 1.33 \\
\hline MT-ND1 & 978 & $476(48.67 \%)$ & 0.194 & 0.066 & 0.531 & 0.125 & 1.64 \\
\hline MT-ND2 & 1041 & $599(57.54 \%)$ & 0.223 & 0.120 & 0.504 & 0.238 & 1.55 \\
\hline MT-ND3 & 352 & $165(46.88 \%)$ & 0.188 & 0.157 & 0.281 & 0.558 & 1.46 \\
\hline MT-ND4 & 1378 & $717(52.03 \%)$ & 0.202 & 0.099 & 0.473 & 0.209 & 1.34 \\
\hline MT-ND4L & 297 & $152(51.18 \%)$ & 0.192 & 0.086 & 0.479 & 0.179 & 1.21 \\
\hline MT-ND5 & 1820 & $984(54.07 \%)$ & 0.209 & 0.112 & 0.478 & 0.235 & 1.35 \\
\hline MT-ND6 & 522 & $308(59.00 \%)$ & 0.217 & 0.268 & 0.232 & 1.151 & 2.72 \\
\hline overall & 11402 & $5483(48.09 \%)$ & 0.189 & & & & \\
\hline
\end{tabular}




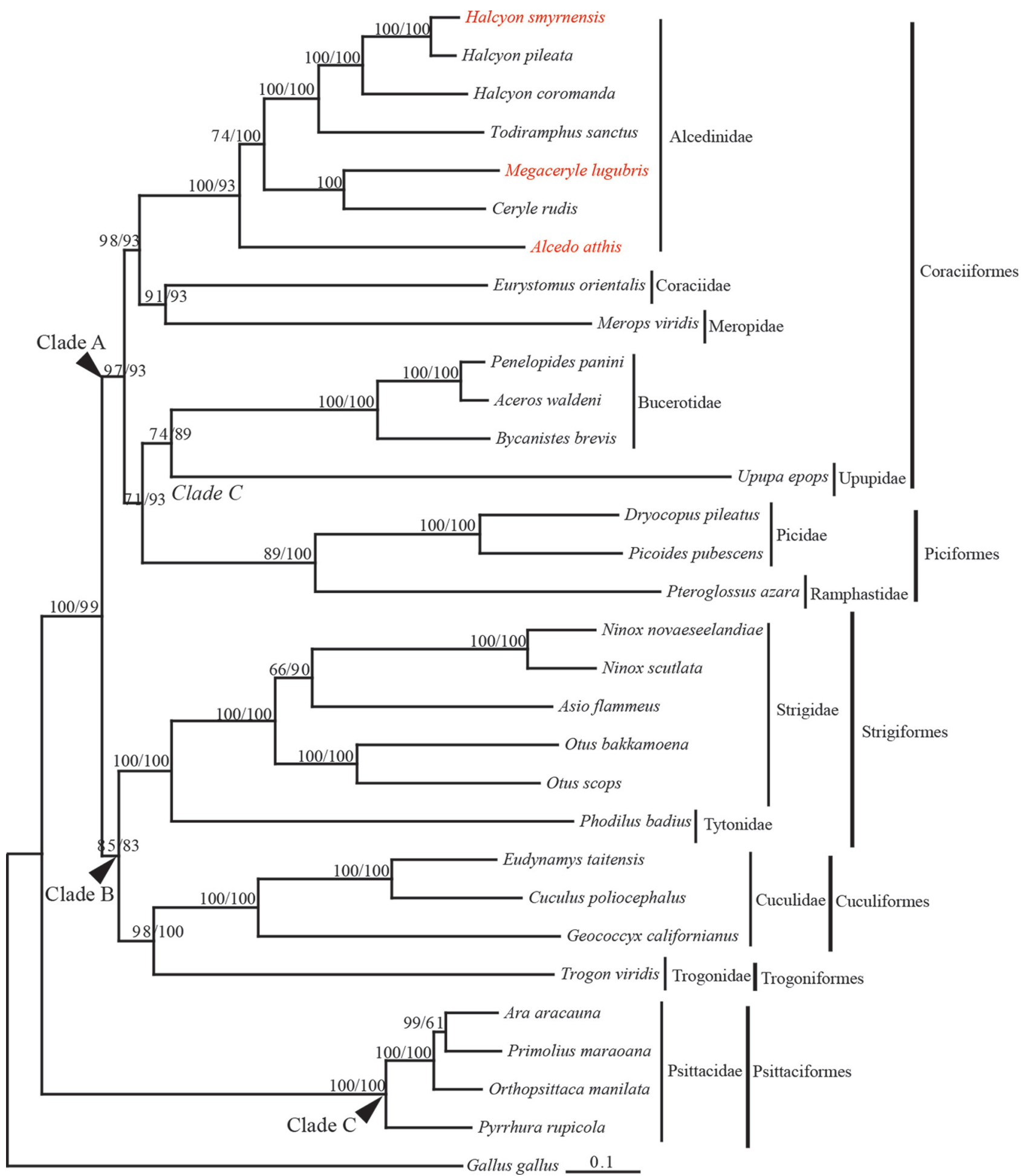

Figure 2 - Phylogenetic tree of 30 species from 12 families of six orders with G. gallus as outgroups. Analyses were based on mitogenome sequences without control regions and the sequences of 12 PCGs without MT-ND6. The Bayesian posterior probability (PP) values for each node were presented. Values for the first set of data were listed in front of those for the second set of data.

groups. Clade $\mathrm{C}$ contains species from Psittacidae, which is the basal clade of the tree.

The relationships among three families of Coraciiformes (Alcedinidae, Coraciidae and Meropidae) displayed in our trees (Figure 2) are congruous with the results of newly published phylogenetic research based on mi- tochondrial DNA markers (Tamashiro et al., 2019). Our tree clearly shows that Bucerotidae and Upupidae are more closely related to Picidae (Piciformes) than to other families of Coraciiformes (Figure 2). Our results support a previous proposal that Bucerotidae and Upupidae should be separated from Coraciiformes, which was inferred from phylogenetic 
studies using morphological characters (Burton, 1984; Olson, 1985; Mayr, 2003;), nuclear DNA (e.g. Sibley and Ahlquist, 1990; Johansson et al., 2001; Hackett et al., 2008; Jetz et al., 2012; Ödeen and Hästad, 2013; Jarvis et al., 2014; Prum et al., 2015; Reddy et al., 2017) and mtDNA (e.g. de los Monteros, 2000; Ericson et al., 2006; Pacheco et al., 2011; Mahmood et al., 2014; Sun et al., 2017; Tamashiro et al., 2019). Our tree also displays that Coraciiformes is more closely related to Picidae (Piciformes) than to Trogonidae (Trogoniformes), which agreed with phylogenetic investigations based on both nuclear DNA (Hackett et al., 2008; Jetz et al., 2012; Ödeen and Hästad, 2013; Jarvis et al., 2014) and mtDNA (Ericson et al., 2006; Tamashiro et al., 2019). However, the relationships among classical Coraciiformes (Alcedinidae, Coraciidae and Meropidae), "Bucerotes" (Bucerotidae and Upupidae) and Picidae (Piciformes) presented in our tree are inconsistent with the results of these studies.

Previous investigations involving different taxa and gene markers (nuclear DNA: Johansson et al., 2001; Hackett et al., 2008; Jarvis et al., 2014; mitochondrial DNA: de los Monteros, 2000; Ericson et al., 2006; Pacheco et al., 2011) did not obtain accordant conclusions on the phylogenetic placement of Trogoniformes. Our results show that, among six orders involved in this study, Trogonidae (Trogoniformes) and Cuculidae (Cuculiformes) are the closest relatives, and that they form a sister taxon to Strigidae (Strigiformes) (Figure 2). In our tree, Psittacidae (Psittaciformes) is the basal clade, indicating distant relationships between Psittaciformes and other five orders (Figure 2). The result is congruous with the conclusion of studies using nuclear (Jetz et al., 2012; Jarvis et al., 2014) and mitochondrial DNA markers (Ericson et al., 2006; Pratt et al., 2009; Tamashiro et al., 2019).

The limitation on taxa in this study would be one of the reasons for inconsistent results between our study and previous researches. More Coraciiform mitogenome data are necessary to resolve the phylogenesis of Coraciiformes and to test the results in previous studies. The new mitogenome data presented in this paper represent a contribution to this long-term goal.

\section{Acknowledgments}

This work was supported by the SecondTibetan Plateau Scientific Expedition and Research program (STEP) (Grant No.2019QZKK0501) and the Natural Scientific Foundation of China (No. 31371252 and 31171189).

\section{Conflict of Interest}

The authors declare no conflict of interest.

\section{Author contributions}

LH conceived the project and drafted the manuscript; MJ, KL and HY performed the experiments; MJ and HY performed data analyses and critically read the manuscript. All authors thoroughly read the manuscript and approved the version to be published.

\section{References}

Abbott CL, Double MC, Trueman JWH, Robinson A and Cockburn A (2005) An unusual source of apparent mitochondrial heteroplasmy: Duplicate mitochondrial control regions in Thalassarche albatrosses. Mol Ecol 14:3605-3613.

Bernt M, Donath A, Jühling F, Externbrink F, Florentz C, Fritzsch G, Pütz J, Middendorf M and Stadler PF (2013) MITOS: Improved de novo metazoan mitochondrial genome annotation. Mol Phylogenet Evol 69:313-319.

Bi D, Ding HW, Wang QQ, Jiang L, Lu WK, Wu X, Zhu R, Zeng JH, Zhou SB, Yang XJ, et al. (2019) Two new mitogenomes of Picidae (Aves, Piciformes): Sequence, structure and phylogenetic analyses. Int J Biol Macromol 133:683-692.

Bi XX, Huang L, Jing MD, Zhang L, Feng PY and Wang AY (2012) The complete mitochondrial genome sequence of the black-capped capuchin (Cebus apella). Genet Mol Biol 35:545-552.

Brinkmann $\mathrm{H}$ and Philippe $\mathrm{H}$ (2008) Animal phylogeny and large-scale sequencing: Progress and pitfalls. J Syst Evol 46:274-286.

Bruxaux J, Gabrielli M, Ashari H, Prys-Jones R, Joseph L, Milá B, Besnard G and Thébaud C (2018) Recovering the evolutionary history of crowned pigeons (Columbidae: Goura): Implications for the biogeography and conservation of New Guinean lowland birds. Mol Phylogenet Evol 120: 248-258.

Burton PJK (1984) Anatomy and evolution of the feeding apparatus in the avian orders Coraciiformes and Piciformes. Bull $\mathrm{Br}$ Mus (Nat Hist) Zool. 476:331-443.

Campbell V and Lapointe FJ (2011) Retrieving a mitogenomic mammal tree using composite taxa. Mol Phylogenet Evol 58:149-156.

Cooper A, Lalueza-Fox C, Anderson S, Rambaut A, Austin J and Ward R (2001) Complete mitochondrial genome sequences of two extinct moas clarify ratite evolution. Nature 409: 704-707.

Cracraft J (1981) Toward a phylogenetic classification of the recent birds of the world (Class Aves). Auk. 98:681-714.

Cracraft J (1988) The major clades of birds. In: M.J. Benton, (ed.) The phylogeny and classification of the tetrapods, vol.1: Amphibians, reptiles, birds, Systematics Association. Oxford University Press, Oxford, pp 339-361.

De Los Monteros AE (2000) Higher-level phylogeny of Trogoniformes. Mol Phylogenet Evol 14:20-34.

De Los Monteros AE (2003) Models of the primary and secondary structure for the 12S rRNA of birds: A guideline for sequence alignment. DNA Seq 14:241-256.

Desjardins P and Morais R (1990) Sequence and gene organization of the chicken mitochondrial genome: A novel gene order in higher vertebrates. J Mol Biol 212:599-634.

Eberhard JR, Wright TF and Bermingham E (2001) Duplication and concerted evolution of the mitochondrial control region in the parrot genus Amazona. Mol Biol Evol 18:1330-1342.

Ericson PGP, Anderson CL, Britton T, Elzanowski A, Johansson US, Källersjö M, Ohlson JI, Parsons TJ, Zuccon D and Mayr G (2006) Diversification of Neoaves: Integration of molecular sequence data and fossils. Biol Lett 2:543-547.

Gibb GC, Kardailsky O, Kimball RT, Braun EL and Penny D (2007) Mitochondrial genomes and avian phylogeny: Complex characters and resolvability without explosive radiations. Mol Biol Evol 24:269-280.

Gibson A, Gowri-Shankar V, Higgs PG and Rattray M (2005) A comprehensive analysis of mammalian mitochondrial ge- 
nome base composition and improved phylogenetic methods. Mol Biol Evol 22:251-264.

Gong J, Zhao RP, Huang QR, Sun XM, Huang L and Jing MD (2017) Two mitogenomes in Gruiformes (Amaurornis akool/A.phoenicurus) and the phylogenetic placement of Rallidae. Genes Genom 39:987-995.

Hackett SJ, Kimball RT, Reddy S, Bowie RCK, Braun EL, Braun MJ, Chojnowski JL, Cox WA, Han KL, Harshman J, et al. (2008) A phylogenomic study of birds reveals their evolutionary history. Science 320:1763-1768.

Harlid A, Janke A and Arnason U (1998) The complete mitochondrial genome of Rhea americana and early avian divergences. J Mol Evol 46:669-679.

He B, Su TJ, Niu ZQ, Zhou ZY, Gu ZY and Huang DY (2019) Characterization of mitochondrial genomes of three Andrena bees (Apoidea: Andrenidae) and insights into the phylogenetics. Int J Biol Macromol 127:118-125.

Höfling E and Alvarenga HMF (2001) Osteology of the shoulder girdle in the Piciformes, Passeriformes and related groups of birds. Zool Anz 240:196-208.

Huang ZH, Tu FY and Ke DH (2017) Complete mitochondrial genome of blue-throated bee-eater Merops viridis (Coraciiformes: Meropidae) with its taxonomic consideration. Pak J Zool 49: 79-84.

Jarvis ED, Mirarab S, Aberer AJ, Li B, Houde P, Li C, Ho SYW, Faircloth BC, Nabholz B, Howard JT, et al. (2014) Whole-genome analyses resolve early branches in the tree of life of modern birds, Science, 346:1320-1331.

Jetz W, Thoma GH, Joy JB, Hartmann K and Mooers AO (2012) The global diversity of birds in space and time. Nature, 491:444-448.

Jiang L, Chen J, Wang P, Ren QQ, Yuan J, Qian CJ, Hua XH, Guo ZC, Zhang L, Yang JK, et al. (2015) The mitochondrial genomes of Aquila fasciata and Buteo lagopus (Aves, Accipitriformes): Sequence, structure and phylogenetic analyses. PloS. One 10:e136297.

Johansson US, Parsons TJ, Irestedt M and Ericson GP (2001) Clades within the 'higher land birds', evaluated by nuclear DNA sequences, J Zool Syst Evol Res 39:37-51.

Junqueiraa ACM, Lessingera AC, Torresa TT, da Silva FR, Vettore AL, Arruda P and Azeredo Espin AML (2004) The mitochondrial genome of the blow fly Chrysomya chloropyga (Diptera: Calliphoridae). Gene 339:7-15.

Kan XZ, Li XF, Lei ZP, Wang M, Chen L, Gao H and Yang ZY (2010) Complete mitochondrial genome of Cabot's tragopan, Tragopan caboti (Galliformes: Phasianidae). Genet Mol Res 9: 1204-1216.

Kan XZ, Li XF, Zhang LQ, Chen L, Qian CJ, Zhang XW and Wang L (2010) Characterization of the complete mitochondrial genome of the Rock pigeon, Columba livia (Columbiformes: Columbidae). Genet Mol Res 9:1234-1249.

Kang H, Li B, Ma X and Xu Y (2018) Evolutionary progression of mitochondrial gene rearrangements and phylogenetic relationships in Strigidae (Strigiformes). Gene 647:8-14.

Kemp A (1995) The Hornbills: Bucerotiformes, Oxford University Press, Oxford, 302 p.

Kumar S, Stecher G, Li M, Knyaz C and Tamura K (2018) MEGA $\mathrm{X}$ : Molecular Evolutionary Genetics Analysis across computing platforms. Mol Biol Evol 35:1547-1549.

Leclercq S, Rivals E and Jarne P (2010) DNA slippage occurs at microsatellite loci without minimal threshold length in humans: a comparative genomic approach. Genome Biol Evol 2:325-335.
Levinson G and Gutman GA (1987) Slipped-strand mispairing: a major mechanism for DNA sequence evolution. Mol Biol Evol 4:203-221.

Li X, Huang Y and Lei F (2015) Comparative mitochondrial genomics and phylogenetic relationships of the Crossoptilon species (Phasianidae, Galliformes). BMC Genomics 16:42.

Li X, Ogoh K, Ohba N, Liang X and Ohmiya Y (2007) Mitochondrial genomes of two luminous beetles, Rhagophthalmus lufengensis and $R$. ohbai (Arthropoda, Insecta, Coleoptera). Gene 392: 196-205.

Lowe TM and Chan PP (2016) tRNAscan-SE On-line: Integrating search and context for analysis of transfer RNA genes. Nucleic Acids Res 44 (W1):W54-W57.

Mahmood MT, McLenachan PA, Gibb GC and Penny D (2014) Phylogenetic position of avian nocturnal and diurnal raptors. Genome Biol Evol 6:326-332.

Maurer DR, Raikow RJ (1981) Appendicular myology, phylogeny, and classification of the avian order Coraciiformes (including Trogoniformes). Ann Carnegie Mus 50:417-434.

Mayr G, Manegold A and Johansson US (2003) Monophyletic groups within 'higher land birds' - comparison of morphological and molecular data. J Zool Syst Evol Res 41:233-248.

Mayr G (2005) The Paleogene Old World potoo Paraprefica Mayr, 1999 (Aves, Nyctibiidae): Its osteology and affinities to the New World Preficinae Olson, 1987. J Syst Palaeontol 3:359-370.

Mindell DP, Sorenson MD and Dimcheff DE (1998) Multiple independent origins of mitochondrial gene order in birds. Proc Natl Acad Sci USA 95:10693-10697.

Mjelle KA, Karlsen BO, Jorgensen TE, Moum T and Johansen SD (2008) Halibut mitochondrial genomes contain extensive heteroplasmic tandem repeat arrays involved in DNA recombination. BMC Genomics 9:10.

Morgan-Richards M, Trewick SA, Bartosch-Härlid A, Kardailsky O, Phillips MJ, McLenachan PA and Penny D (2008) Bird evolution: Testing the Metaves clade with six new mitochondrial genomes. BMC Evol Biol 8:20.

Nabholz B, Jarvis ED and Ellegren H (2010) Obtaining mtDNA genomes from next-generation transcriptome sequencing: A case study on the basal Passerida (Aves: Passeriformes) phylogeny. Mol Phylogenet Evol 57:466-470.

Ödeen A and Hästad O (2013) The phylogenetic distribution of ultraviolet sensitivity in birds. BMC Evol Biol 13:36.

Ojala D, Montoya J and Attardi G (1981) tRNA punctuation model of RNA processing in human mitochondrial. Nature 290:470-474.

Olson SL (1985) The fossil record of birds. In: Farner DS, KInng JR and Parkes KC (eds) Avian Biology, Academic Press, New York, pp 79-252.

Pacheco MA, Battistuzzi FU, Lentino M, Aguilar RF, Kumar S and Escalante AA (2011) Evolution of modern birds revealed by mitogenomics: Timing the radiation and origin of major orders. Mol Biol Evol 28:1927-1942.

Paton T, Haddrath O and Baker AJ (2002) Complete mitochondrial DNA genome sequences show that modern birds are not descended from transitional shorebirds. Proc R Soc Lond B Biol Sci 269: 839-846.

Piganeau G, Gardner M and Eyre-Walker A (2004) A broad survey of recombination in animal mitochondria. Mol Biol Evol 21:2319-2325.

Posada D and Crandall KA (1998) Modeltest: Testing the model of DNA substitution. Bioinformatics 14:817-818.

Pratt RC, Gibb GC, Morgan-Richards M, Phillips MJ, Hendy MD and Penny D (2009) Toward resolving deep neoaves phylog- 
eny: Data, signal enhancement, and priors. Mol Biol Evol 26: 313-326.

Prum RO, Berv JS, Dornburg A, Field DJ, Townsend JP, Lemmon EM and Lemmon AR (2015) A comprehensive phylogeny of birds (Aves) using targeted next-generation DNA sequencing, Nature, 526:569-573.

Reddy S, Kimball RT, Pandey A, Hosner PA, Braun MJ, Hackett SJ, Han KL, Harshman J, Huddleston CJ, Kingston S, et al. (2017) Why do phylogenomic data dets yield conflicting trees? Data type influences the avian tree of life more than taxon sampling. Syst Biol 66:857-879.

Ren QQ, Li XF, Yuan J, Chen DS, Zhang L, Guo WW, Jiang L, Wang P and Kan XZ (2014) Complete mitochondrial genome of the Blue Eared Pheasant, Crossoptilon auritum (Galliformes: Phasianidae). Mitochondrial DNA A 27:615-617.

Ronquist F and Huelsenbeck JP (2003) MrBayes 3: Bayesian phylogenetic inference under mixed models. Bioinformatics 19:1572-1574.

Sammler S, Bleidorn C and Tiedemann R (2011) Full mitochondrial genome sequences of two endemic Philippine hornbill species (Aves: Bucerotidae) provide evidence for pervasive mitochondrial DNA recombination. BMC Genomics 12:35.

Sbisà E, Tanzariello F, Reyes A, Pesole G and Saccone C (1997) Mammalian mitochondrial D-loop region structural analysis: Identification of new conserved sequences and their functional and evolutionary implication. Gene (Amsterdam) 205:125-140.

Shi W, Kong XY, Wang ZM, Yu SS, Chen HX and De Stasio EA (2013) Pause-melting misalignment: A novel model for the birth and motif indel of tandem repeats in the mitochondrial genome. BMC Genomics 14:103.

Sibley CG and Ahlquist JE (1990) Phylogeny and classification of birds: A study in molecular evolution, Yale University Press, New Haven and London, 976 p.

Sibley CG and Monroe BL (1990) Distribution and taxonomy of birds of the world, Yale University Press, New Haven and London, $108 \mathrm{p}$

Singh TR, Shneor O and Huchon D (2008) Bird mitochondrial gene order: Insight from 3 warbler mitochondrial genomes. Mol Biol Evol 25:475-477.

Slack KE, Janke A, Penny D and Arnason U (2003) Two new avian mitochondrial genomes (penguin and goose) and a summary of bird and reptile mitogenomic features. Gene 302: 43-52.

Song XH, Huang J, Yan CC, Xu GW, Zhang XY and Yue BS (2015) The complete mitochondrial genome of Accipiter virgatus and evolutionary history of the pseudo-control regions in Falconiformes. Biochem Syst Ecol 58:75-84.

Sorenson MD, Ast JC, Dimcheff DE, Yuri T and Mindell DP (1999) Primers for a PCR-based approach to mitochondrial genome sequencing in birds and other vertebrates. Mol Phylogenet Evol 12:105-114.

Sun XM, Zhao RP, Zhang T, Gong J, Jing MD and Huang L (2017) The mitochondrial genomes in Alcedinidae (Ceryle rudis/ Halcyon pileate) and the phylogenetic placement of Coraciiformes. Genetica 145:431-440.

Tamashiro RA, White ND, Braun MJ, Faircloth BC, Braun EL and Kimball RT (2019) What are the roles of taxon sampling and model fit in tests of cyto-nuclear discordance using avian mitogenomic data? Mol Phylogenet Evol 130:132-142.

Toews DPL and Brelsford A (2012) The biogeography of mitochondrial and nuclear discordance in animals. Mol Ecol, 21:3907-3930
Verkuil YI, Piersma T and Baker AJ (2010) A novel mitochondrial gene order in shorebirds (Scolopacidae, Charadriiformes). Mol Phylogenet Evol 57:411-416.

Wetmore A (1960) A classification for the birds of the world, Smithson. Misc Coll 139:1-37.

Yang R, Wu X, Yan P, Su X and Yang B (2010) Complete mitochondrial genome of Otis tarda (Gruiformes: Otididae) and phylogeny of Gruiformes inferred from mitochondrial DNA sequences. Mol Biol Rep 37:3057-3066.

Zhang L, Wang L, Gowda V, Wang M, Li X and Kan X (2012) The mitochondrial genome of the Cinnamon Bittern, Ixobrychus cinnamomeus (Pelecaniformes: Ardeidae): Sequence, structure and phylogenetic analysis. Mol Biol Rep 39:8315-8326.

Zhao Z (2001) Handbook of the birds in China. Jilin Science and Technology Publishing House, Changchun, vol. 1.

Zhong D, Zhao GJ, Zhang ZS and Xun AL (2002) Advance in the entire balance and local unbalance of base distribution in genome. Hereditas 24:351-355.

Zhou X, Lin Q, Fang W and Chen X (2014) The complete mitochondrial genomes of sixteen ardeid birds revealing the evolutionary process of the gene rearrangements. BMC Genomics 15:573.

Zou Y, Jing MD, Bi XX, Zhang T and Huang L (2015) The complete mitochondrial genome sequence of the little egret (Egretta garzetta). Genet Mol Biol 38:162-172.

\section{Supplementary material}

The following online material is available for this article:

Table S1 - Species involved in phylogeny analyses of this study.

Table S2 - Organization of mitogenomes of the A. atthis, $H$. smyrnensis and M. lugubris

Table S3 - Genomic characteristics of 13 coraciiform mtDNA

Table S4 - Sequences of conserved motifs in control regions of A. atthis, H. smyrnensis and M. lugubris

Table S5 - Lengths and base compositions for protein coding genes in mitogenomes of $A$. atthis/H. smyrnensis/M. lugubris

Table S6 Nucleotide compositions in three codon positions of 12 mitochondrial protein coding genes.

Figure S1 - Structure diagram of control region (CR) in mitogenomes of A. atthis, H. smyrnensis and M. lugubris.

Figure S2 - Secondary structures of the 22 tRNAs of three species.

Figure S3 - Predicted secondary structures of MT-RNR1 in A. atthis.

Figure S4 - Predicted secondary structures of MT-RNR2 in A. atthis.

Figure S5 - Relative synonymous codon usage (RSCU) in the mitogenome of A. atthis, H. smyrnensis and M. lugubris.

Figure S6 - Alignment of the mitochondrial MT-ND3 gene region spanning the extra base site (designated by $*$ ) for 13 species.

\section{Associate Editor: Alexandro Rodrigues Caetano}

License information: This is an open-access article distributed under the terms of the Creative Commons Attribution License (type CC-BY), which permits unrestricted use, distribution and reproduction in any medium, provided the original article is properly cited. 